\title{
Development of Motion Analysis Tool for Orthopedic diseases
}

\author{
Pavel Jičinský \\ Department of Process Control \\ Faculty of Electrical Engineering and Informatics, University of \\ Pardubice \\ Pardubice, Czech Republic \\ pavel.jicinsky@student.upce.cz
}

\author{
Jan Mareš \\ Department of Process Control \\ Faculty of Electrical Engineering and Informatics, University of \\ Pardubice \\ Pardubice, Czech Republic \\ Jan.Mares@vscht.cz
}

\section{ANALOG FRONT-END DESIGN}

devices for diagnostics from motion data. Such devices can be used in a wide range of medical disciplines, from rehabilitation medicine to neurology. In this case we focus on rehabilitation after surgery of large joints (shoulder, knee, hip). In this article, we present the basic prototype of devices for collecting motion data and their fusion with electromyographic data, from which we will then analyze the disturbances of momentum.

\section{Keywords—EMG, orthopedic, biopotential, measurements}

\section{INTRODUCTION}

Large joint surgery (especially hip) is a major intervention in the patient's quality of life. After its completion, a long rehabilitation is necessary, the success of which strongly depends on the subjective assessment of progress by a doctor and a physiotherapist. This article deals with an innovative approach to the analysis of movement disorders in patients after this type of surgery.

The aim of the work is to create a complex mobile device (which will have a patient on the body) that senses the movements of the lower limbs and the EMG signal from selected muscles. By merging and subsequent analysis of these data, it will be possible to objectively assess the current state of the rehabilitating patient.

\section{STATE OF THE ART}

Gait and movement analysis are a hot topic and many research groups deal with it from both the medicinal and signal processing point of view.

From a medical point of view, motion analysis can be used to diagnose a large number of neurological diseases [1] [2], sleep apnea [3] [4]. Recently, several publications can be found, where the authors describe the use of movement analysis in rehabilitation [5] especially of patients after head and neck surgery (mimetic momentum) [6]. Rehabilitation after orthopedic surgery is therefore naturally a topic that should also be explored. However, in the literature, it stays in the background compared to other topics.

From the point of view of signal processing, this topic is also interesting and current. In the past, the Fourier transform, or the Wavelet transform have been successfully used to analyze such data. Currently, research is moving towards neural networks, deep learning, etc.
Designing an analog front-end for measurements of biopotentials is a complex task as it requires the designer to be aware of potential problems.

\section{A. Design considerations}

First problem is that the measuring device is in direct contact with the patient's body for prolonged periods of time and it must provide an extraordinary amount of protection for the patient. The real problem here is the electric insulation and the electromagnetic interference. It is common to see the cheapest analog front ends (AFE) available being powered directly from an USB cable providing power supply and the data interface at the same time. These devices inherit a fatal design flaw in a form of lack of the galvanic isolation and the high frequency interference from the USB data transmission. Most of the available solutions deal with this problem using wireless data transmission using $\mathrm{Wi}-\mathrm{Fi}$ or Bluetooth while being battery powered [7] [8] [9]. This effectively severes the ground coupling. It however also increases demands for the battery capacity in the systems using $\mathrm{Wi}-\mathrm{Fi}$, or it limits the data throughput in the ones using Bluetooth or similar low data throughput technologies. Another way of dealing with the galvanic isolation is use of isolators. The communication interface is decoupled using such a device resulting in a separated power supply domain for upstream and downstream parts of the circuitry. The AFE board could then be powered using batteries or a switched mode power supply (SMPS) with galvanic insulation powered directly from the USB interface could be used. Great caution should be taken however when using any kind of switched mode power supplies for powering such a delicate circuit as AD converter. This problem is crucial especially when the switching frequency of the power supply falls into the Nyquist bandwidth of the sampling circuit of the ADC [10]. This power supply referred interference can be mitigated by using a low noise SMPS, by choosing an appropriate ADC topology and by proper noise decoupling of the power supply rails.

Great care should be taken when designing an electrical device connected directly to a human being. Biopotential measurement devices usually measure electrical signals resulting from bioactivity. These signals' amplitudes typically range from tens of microvolts (EEG) to a couple of millivolts (ECG, EMG). For EMG these signals originate from the muscle motor units firing in order to move a skeletal muscle [11]. The subject's muscle can be modeled as a voltage source with high series resistance. A probe cable adds to this resistance too as it is connected in series with this 
source. To counter this high series resistance a several order higher input resistance amplifiers are used for biopotential data acquisition. This high enough input resistance limits the amount of potentially harmful current flowing through the patient's body, but it also makes the input more susceptible to interference. The IEC standard for Medical electrical equipment IEC60601 defines a limit for a maximum amount of leakage current allowed through the patient's body to 50 $\mu \mathrm{A}$ [12]. With the input impedance of the modern analog front-end amplifiers in the order of Megaohms, this criterion is fulfilled with almost no trade-offs. The input impedance definition of the AFE is problematic however as it is typically defined only under normal operational conditions. The voltage measurement devices such as AFEs are often connected to an unknown circuit and could be exposed to a voltage level far surpassing its designed ratings. The IEC60601 standard also demands the medical devices to be able to withstand a high voltage surge without any safety compromises. This applies especially for the wearable devices in direct contact with patient's body should he or she undergo a defibrillation.

\section{B. Input and Analog circuitry}

The ADS1298 [13] delta-sigma ADC was chosen as the front-end IC for this project. It integrates 8 channel simultaneous sampling 24-bit delta sigma converter. The clock signal for this circuit is provided internally with a frequency of $2.048 \mathrm{MHz}\left(\mathrm{f}_{\mathrm{MOD}}\right)$. The converter samples the input signal at a rate equal to $\mathrm{f}_{\mathrm{MOD}} / 4$ in a high-resolution mode. This sampled signal is then further decimated by a factor of 256 which results in an output sampling rate of 2 kSPS. The oversampling of the input signal and the subsequent decimation allow using a very simple input antialiasing filters as they do not have to have a high roll-off rate and a simple first order RC filter is usually enough. This $\mathrm{RC}$ filter is often designed such that the resistor serves also as the current limiter to fulfill the maximum allowable current.

Gel surface electrodes were chosen as a patient/AFE interface as they are relatively cheap and offer an acceptable amount of comfort for patient during prolonged measurement sessions. The electrodes used in this project come with a banana cable connection piece for a quick cable attachment. The banana connector is soldered to a twisted-pair microphone cable TAS-C112 [14] and the cable is secured on the subject's body using adhesive tape to prevent excessive motion artifacts. This cable was chosen for its well-defined parasitic characteristics and a solderable copper spiral wound shielding. The cable shielding proved to be a key aspect of input signal conditioning as well as maintaining a good connector contact resistance. The shielding uses active drive from the Right leg drive (RLD) circuit. Other shielding options were tested but the RLD driver proved to be the best in interference suppression. The inputs are arranged in a differential signal mode and fed into a first order differential $\mathrm{RC}$ filter. The input stage circuit is shown in figure 1.

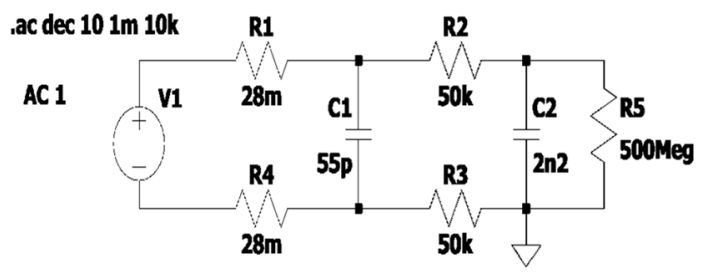

Fig. 1. Input circuit

The input stage of the used AFE provide many ways to detect a lead-off condition when the electrode comes loose which hinders the contact conductivity and thus reduce the signal quality. In this project the low but well-defined current source/sinks with the current of $6 \mathrm{nA}$ were used for this purpose. The AFE integrates these current sources which are connected to the both differential inputs, the positive to the current source and the negative to the current sink. This way, whenever the contact resistance is higher than a defined threshold as a result of bad electrode contact, the AFE detects this and reports this state. This useful feature comes with a drawback however as it effectively halves the input impedance to around $500 \mathrm{M} \Omega$. The $-3 \mathrm{~dB}$ frequency of this filter is around $730 \mathrm{~Hz}$ which covers the frequency bandwidths for the EMG and ECG measurements.

Another crucial feature of the used AFE is the RLD circuit which serves two purposes. First, it creates a defined voltage bias for the input stage amplifiers, ensuring that the input signal stays well within the common mode voltage range. This has two main effects as it forces the input signal to stay between the supply rails of the ADC, avoiding saturation and it also keeps the input stage amplifiers in the recommended operation ranges and in turn maintaining the high input impedance. The seconds purpose is to reduce the input signal noise by a negative feedback loop. The RLD output is then fed through the protection resistor to the patient's body. The Analog input circuitry is shown in figure 2 .

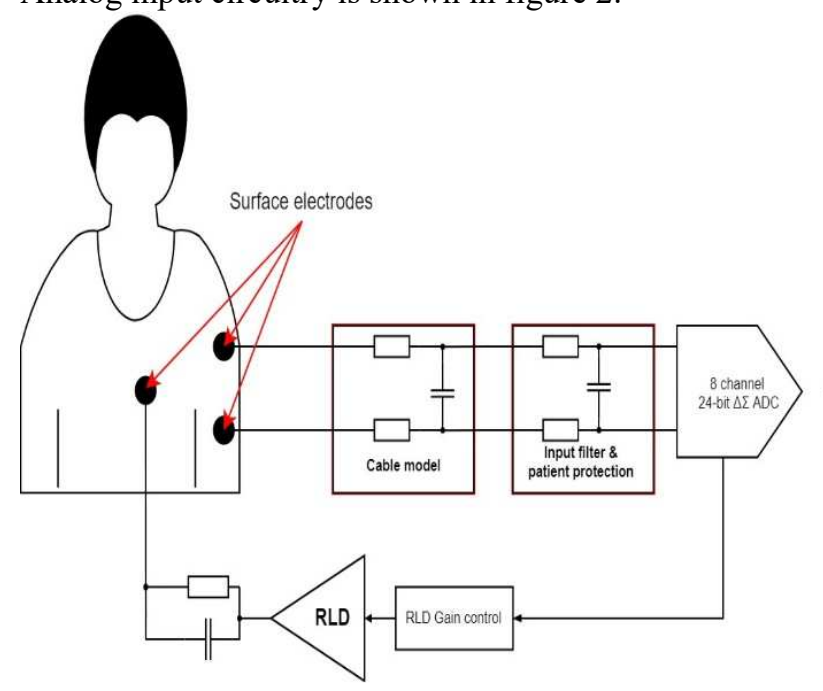

Fig. 2. Analog input stage

The reference voltage for the AFE is driven internally and its value is $2.4 \mathrm{~V}$. It is possible to connect an external voltage reference source to obtain even greater reference accuracy. The datasheet however does not state the absolute limits for this supply. With the 24-bit two's complement output data 
and 2.4 V reference voltage, the LSB is approximately 0.286 $\mu \mathrm{V}$ according to (1).

$$
L S B_{[V]}=\frac{V_{R e f}}{2^{23}}
$$

When comparing the LSB value with the expected amplitudes of biopotential signals it is clear that the actual number of bits containing any information about input signal will be significantly lower than the advertised resolution. This can be countered by preamplification of the input signal. The ADS1298 integrates a low noise PGA with up to $12 x$ gain, resulting in an additional 3 to 4 bits. An external preamplification can be also staged to the input chain, but its parasitic characteristics cannot generally beat the integrated solution. It would also make the lead-off detection useless as the lead-off circuitry would drive the output stage of the preamplifier rather than the patient's body. The solution with integrated PGA turned out to be sufficient in providing decent measurements and maintaining the general shape of the signal.

\section{Power supplies and Auxiliary circuitry}

The AFE designed for this project uses a $3 \mathrm{Ni}-\mathrm{MH}$ cells as the main power supply. It can also be powered through the isolated DC/DC converter IC B0505S-1W. The input voltage is fed into an LDO regulator TPS7A9101 with an output voltage set by a resistive divider to $3.3 \mathrm{~V}$. The output of this LDO is connected to a dual LDO/Charge pump inverter IC LM27762 providing a bipolar power supply for the analog front-end IC. The output voltage is defined again by a resistive divider and is finely trimmed to $\pm 1.8 \mathrm{~V}$. The power supply chain is illustrated in figure 3 .

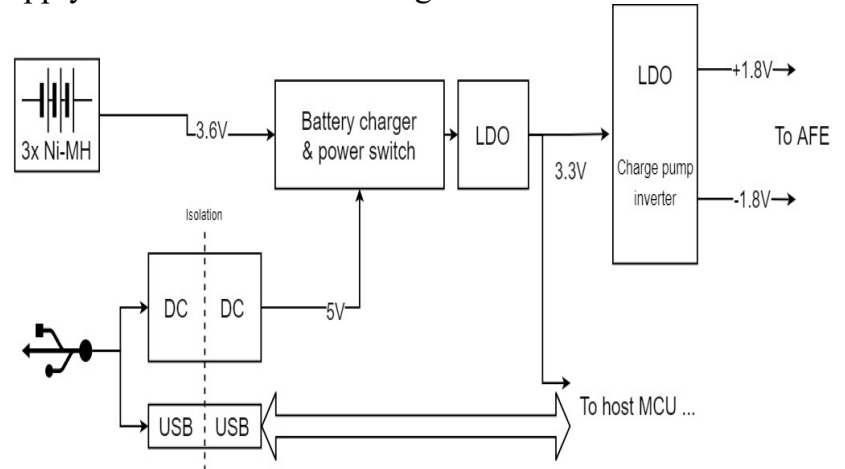

Fig. 3. Power supply chain

The designed PCB uses separated ground planes for digital and analog domains. The ground planes were designed according to the star grounds rule.

\section{Data Interface and Control logic}

The used AFE IC is interfaced using SPI and 5 additional GPIO pins. The STM32F405 MCU was chosen as the main microcontroller for this project as it integrates full speed USB port and is relatively cheap and available. The USB interface is connected through the ADuM4160 isolator to the PC running a test bench application via standard USB cable type B. The MCU uses DMA transfers for both the SPI and USBCDC communications and it transmits a packet of $64 \times 8$ values (for 8 channels) to the PC application. The default AFE settings for this project if not stated otherwise is $2 \mathrm{kSPS}$ sampling rate and $12 \mathrm{x}$ gain for each channel. The wired connection is used to overcome difficulties with the lack of throughput with wireless solutions. It does limit the test subject's range of motion, but it is still very usable when testing using treadmill or exercise bike.

\section{MEASURED DATA}

A PC application written in C\# was created for this project as a test bench application. It uses virtual COM ports to communicate with the AFE using USB. It allows a realtime charting of all the 8 channels simultaneously, although with a moderate performance hits. It also allows spectral analysis for each channel with additional metrics, e.g. RMS and standard deviation of the input signal. The input signals for each channels are filtered using elliptic $4^{\text {th }}$ order high-pass IIR filter in order to remove significant input DC offset. It also helps removing the cable movement glitches that are present in the surface mount electrodes measurements. The passband frequency is $10 \mathrm{~Hz}$ with $80 \mathrm{~dB}$ stopband attenuation. The filter was designed using Matlab filterDesigner tool and converted into direct form I using single filter section as is required by the used C\# DSP library MathNET [15].

The input stage of the designed AFE was put to a test for input referred noise values. The datasheet contains a table for each individual sampling settings and the resulting noise characteristics. The measured values are summarized in table 1.

TABLE I. INPUT REFERRED NOISE MEASUREMENTS

\begin{tabular}{|c|c|c|c|c|c|c|c|}
\hline \multicolumn{2}{|c|}{ Gain 1} & \multicolumn{2}{|c|}{ Gain 2} & \multicolumn{2}{|c|}{ Gain 3} & \multicolumn{2}{|c|}{ Gain 4} \\
\hline $\begin{array}{c}\mathbf{f}_{\mathbf{S}} \\
\text { [SPS] }\end{array}$ & $\begin{array}{c}\text { Noise } \\
\text { RMS } \\
{[\mu \mathrm{V}]}\end{array}$ & $\begin{array}{c}\mathbf{f}_{\mathrm{S}} \\
\text { [SPS] }\end{array}$ & $\begin{array}{l}\text { Noise } \\
\text { RMS } \\
{[\mu \mathrm{V}]}\end{array}$ & $\begin{array}{c}\mathbf{f}_{\mathrm{S}} \\
\text { [SPS] }\end{array}$ & $\begin{array}{c}\text { Noise } \\
\text { RMS } \\
{[\mu \mathrm{V}]}\end{array}$ & $\begin{array}{c}\mathbf{f}_{\mathbf{s}} \\
{[\mathbf{S P S}]}\end{array}$ & $\begin{array}{c}\text { Noise } \\
\text { RMS } \\
{[\mu \mathrm{V}]}\end{array}$ \\
\hline 500 & 2,04 & 500 & 1,15 & 500 & 0,84 & 500 & 0,64 \\
\hline 1000 & 2,81 & 1000 & 1,64 & 1000 & 1,19 & 1000 & 0,89 \\
\hline 2000 & 4,08 & 2000 & 2,23 & 2000 & 1,61 & 2000 & 1,30 \\
\hline 4000 & 6,05 & 4000 & 3,21 & 4000 & 2,28 & 4000 & 1,92 \\
\hline 8000 & 11,99 & 8000 & 6,29 & 8000 & 4,33 & 8000 & 3,45 \\
\hline \multicolumn{2}{|c|}{ Gain 6} & \multicolumn{2}{|c|}{ Gain 8} & \multicolumn{2}{|c|}{ Gain 12} & & \\
\hline $\begin{array}{c}\mathbf{f}_{\mathbf{S}} \\
\text { [SPS] }\end{array}$ & $\begin{array}{c}\text { Noise } \\
\text { RMS } \\
{[\mu \mathrm{V}]}\end{array}$ & $\begin{array}{c}\mathbf{f s}_{\mathbf{S}} \\
\text { [SPS] }\end{array}$ & $\begin{array}{l}\text { Noise } \\
\text { RMS } \\
{[\mu \mathrm{V}]}\end{array}$ & $\begin{array}{c}\mathbf{f}_{\mathrm{S}} \\
{[\mathbf{S P S}]}\end{array}$ & $\begin{array}{l}\text { Noise } \\
\text { RMS } \\
{[\mu \mathrm{V}]}\end{array}$ & & \\
\hline 500 & 0,56 & 500 & 0,48 & 500 & 0,45 & & \\
\hline 1000 & 0,72 & 1000 & 0,63 & 1000 & 0,62 & & \\
\hline 2000 & 1,05 & 2000 & 0,93 & 2000 & 0,82 & & \\
\hline 4000 & 1,51 & 4000 & 1,32 & 4000 & 1,15 & & \\
\hline 8000 & 2,51 & 8000 & 1,99 & 8000 & 1,78 & & \\
\hline
\end{tabular}

The RMS value of the input noise was calculated for 2048 values for each channel. These 8 values were then averaged. The input channels were set to be shorted internally - a feature used exactly for a purpose of input noise measurements. It is clear from the table 1 outputs that the measured noise levels are the same or even smaller than those 
published in the datasheet. Screenshot from the input noise measurements is depicted in figure 4.

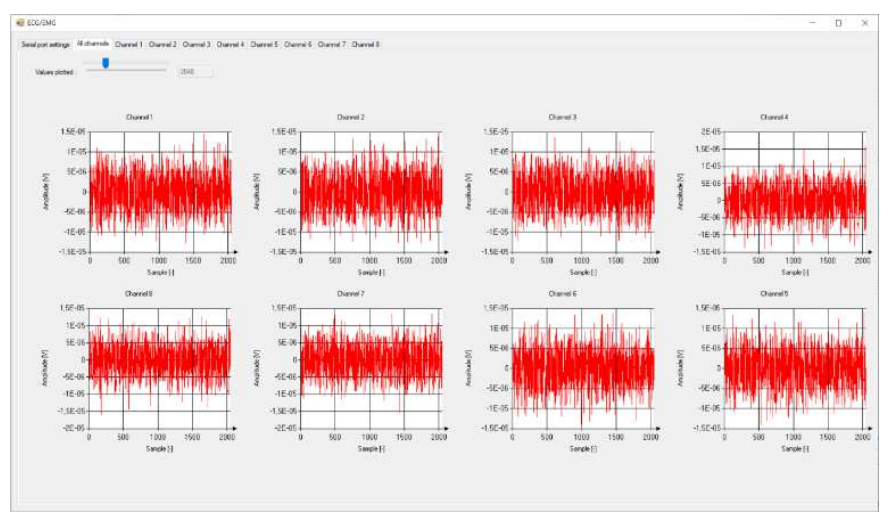

Fig. 4. Input noise measurements ( 8 channels $-1 x$ Gain)

DC offset was clearly filtered out as is apparent from figure 4. Without this filtering, the input offset has a several orders of magnitude larger absolute value than the amplitude of the signal of interest and is always dominant in the resulting charts. The application is capable of plotting more than 16384 samples for each channel in the real time, although at a cost of user responsiveness.

The figure 5 shows the effect of the USB isolator on 1024 samples of the input signal taken from the test subject's biceps. The top part of the chart represent the channel 1 samples taken with gain settings of $12 x$ without USB isolation. The bottom part represents the same data but with USB interface isolated. The test subject had his feet in direct contanct with the ground without any isolation. Multiples of $50 \mathrm{~Hz}$ mains frequency (in Czech Republic) are clearly visible in the top chart in contrast with the bottom chart where the higher harmonics are attenuated.
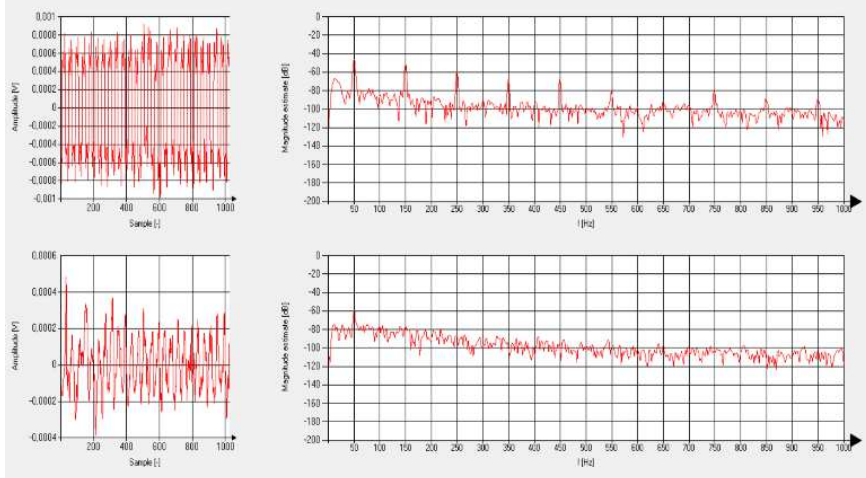

Fig. 5. Input signal without and with USB isolation (12x Gain)

The biceps was held at rest hanging down during the signal acquisition and the $\mathrm{Y}$ axis was zoomed in in order to magnify the effect of noise on the sampled signal.

For the following measurements the input data were filtered using the same $4^{\text {th }}$ order higpass filter with cutoff frequency of $10 \mathrm{~Hz}$ coupled with a $10^{\text {th }}$ order elliptic bandstop IIR filter with stopband of 45 to $55 \mathrm{~Hz}, 80 \mathrm{~dB}$ attenuation and $0.5 \mathrm{~dB}$ ripple in the both passbands.

The AFE's capability of ECG cardiogram acquisition was also tested, although it is not the main purpose of this project. The cardiogram is depicted in figure 6 .

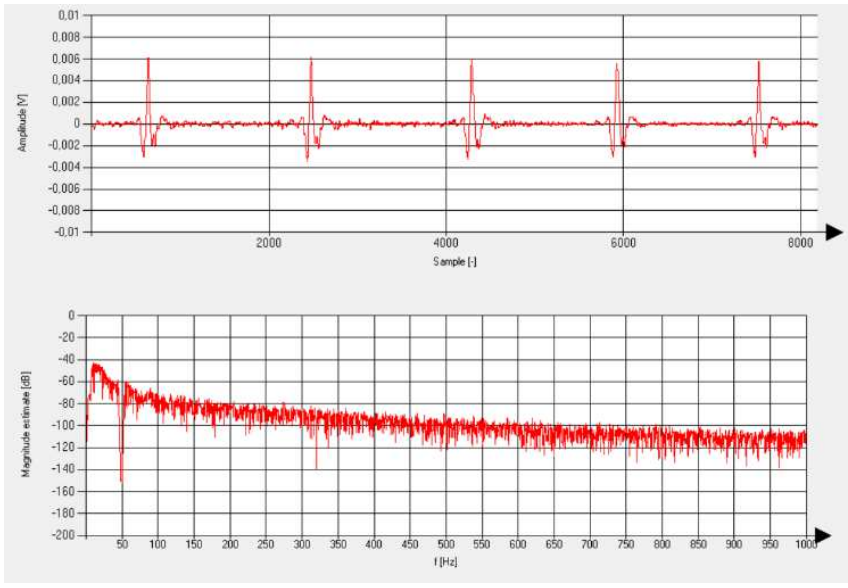

Fig. 6. ECG measurements

The effect of the filtering stage is clearly visible in the magnitude estimate chart - DC signal attenuated below -120 $\mathrm{dB}$ and $80 \mathrm{~dB}$ drop around the $50 \mathrm{~Hz}$ mains frequency. Even better filtering could be achievable, it would require a significant changes in the MathNet filtering library however as it only works with single stage filter coefficients. It has proven diffucult to find a stable IIR filter using these settings, but bulding it using biquadtratic sections proved to be almost always stable.

\section{CONCLUSION}

The aim of this paper is to describe the initialization phase of a project focused on the analysis of lower limb movements and the fusion of this data with EMG records. The article mainly describes the development of equipment and ensuring communication and data acquisition.

After testing, the device will be handed over to an Clinic of Orthopedy, University Hospital Kralovské Vinohrady Prague, where data acquisition from real patients will take place

\section{FUTURE WORK}

The constructed analog front-end served as a proof of concept test bench for EMG and ECG measurements. It will be used as a part of a complex device for gait analysis and diagnosis of movement disorders in orthopedic patients. The AFE will monitor the skeletal muscle activity during rehabilitation process after large joint surgeries as well as during patient's examinations. The system will contain additional circuitry for measuring and displaying spatial data from selected joints of patient under examination using inertial measurement units (IMU). Obtained spatial data in form of linear and radial accelerations as well as orientation relative to Eath's magnetic field will be fused together with the EMG signals of adjacent selected skeletal muscles in order to create a complex 3D model of patient's lower body extermities during movement.

\section{ACKNOWLEDGMENT}

This research was funded by an SGS grant from the Faculty of Electrical Engineering and Informatics, University of Pardubice, Czech Republic. This support is very gratefully acknowledged. 


\section{REFERENCE}

[1] A. Procházka, O. Vyšata, H. Charvátová and M. Vališ, "Motion Symmetry Evaluation Using Accelerometers and Energy Distribution", Symmetry, vol. 11, no. 7, 2019.

[2] L. Oudre, R. Barrois-Müller, T. Moreau, C. Truong, A. Vienne-Jumeau, D. Ricard, N. Vayatis and P. Vidal, "Template-Based Step Detection with Inertial Measurement Units", Sensors, vol. 18, no. 11, 2018.

[3] M. Schätz, A. Procházka, J. Kuchyňka and O. Vyšata, "Sleep Apnea Detection with Polysomnography and Depth Sensors", Sensors, vol. 20, no. 5, 2020.

[4] M. Schatz, F. Centonze, J. Kuchynka, O. Tupa, O. Vysata, O. Geman and A. Prochazka, "Statistical recognition of breathing by MS Kinect depth sensor", in 2015 International Workshop on Computational Intelligence for Multimedia Understanding (IWCIM), 2015, pp. 1-4.

[5] R. Nussbaum, C. Kelly, E. Quinby, A. Mac, B. Parmanto and B. Dicianno, "Systematic Review of Mobile Health Applications in Rehabilitation", Archives of Physical Medicine and Rehabilitation, vol. 100, no. 1, pp. 115-127, 2019.

[6] J. Kohout, L. Verešpejová, P. Kříž, L. Červená, K. Štícha, J. Crha, K. Trnková, M. Chovanec and J. Mareš, "Advanced Statistical Analysis of 3D Kinect Data: Mimetic Muscle Rehabilitation Following Head and Neck Surgeries Causing Facial Paresis", Sensors, vol. 21, no. 1, 2021.

[7] K. Petkos, S. Koutsoftidis, T. Guiho, P. Degenaar, A. Jackson, S. Greenwald, P. Brown, T. Denison and E. Drakakis, "A high-performance $8 \mathrm{nV} / \sqrt{\mathrm{Hz}} 8$-channel wearable and wireless system for real-time monitoring of bioelectrical signals". 2019.

[8] D. Piccinini, N. Andino, S. Ponce, M. Roberti and N. López, "Wearable System for Acquisition and Monitoring of Biological Signals". 2016.

[9] B. Babusiak, S. Borik and M. Smondrk, "TwoElectrode ECG for Ambulatory Monitoring with Minimal Hardware Complexity". 2020.

[10] R. Du, "AN-1141 - Powering a Dual Supply Precision ADC with Switching Regulators", 2012. [Online]. Available: https://www.analog.com/media/en/technicaldocumentation/application-notes/AN-1141.pdf. [Accessed: 2021].

[11] A. Vigotsky, I. Halperin, G. Lehman, G. Trajano and T. Vieira, "Interpreting Signal Amplitudes in Surface Electromyography Studies in Sport and Rehabilitation Sciences". 2018.

[12] "IEC 60601-1-11:2015", iso.org. [Online]. Available: https://www.iso.org/standard/65529.html. [Accessed: 2021].

[13] "ADS1298 Datasheet", ti.com. [Online]. Available: https://www.ti.com/lit/ds/symlink/ads1294.pdf?ts=16 $16171172767 \&$ ref_url $=$ https $\% 253 \mathrm{~A} \% 252 \mathrm{~F} \% 252 \mathrm{Fww}$ w.google.com $\% 25 \overline{2} \mathrm{~F}$.
[14] "Technical Datasheet C112 - 2x0,35 mm", tme.eu. [Online]. Available: https://www.tme.eu/Document/1dc828ad49ff926c63f 748ec1268ecaa/C112.pdf.

[15] "Math.NET", Numerics. [Online]. Available: https://numerics.mathdotnet.com/. 